Ann-Christin Pecher: None declared, Daniela Dörfel: None declared, Jörg Henes Grant/research support from: Novartis, Roche-Chugai, Consultant of: Novartis, Roche, Celgene, Pfizer, Abbvie, Sanofi, Boehringer-Ingelheim,

DOI: 10.1136/annrheumdis-2020-eular.4296

\begin{tabular}{|l|l}
\hline FRI0016 & R835, A NOVEL IRAK1/4 DUAL INHIBITOR IN CLINICAL \\
DEVELOPMENT, BLOCKS TOLL-LIKE RECEPTOR 4 \\
(TLR4) SIGNALING IN HUMAN AND MOUSE
\end{tabular}

C. Lamagna ${ }^{1}$, C. Gundel ${ }^{1}$, M. Chan ${ }^{1}$, C. Young ${ }^{1}$, S. Braselmann ${ }^{1}$, R. Frances ${ }^{1}$, S. Yi ${ }^{1}$, Y. Chen ${ }^{1}$, G. Park ${ }^{1}$, L. Chou ${ }^{1}$, E. Masuda ${ }^{1}$, V. Taylor ${ }^{1} .{ }^{1}$ Rigel Pharmaceuticals, South San Francisco, United States of America

Background: Toll-Like Receptors (TLR) and Interleukin-1 Receptors (IL-1R) play a critical role in the innate immune response as microbial sensors, providing a bridge between the innate and adaptive immunity (1). Interleukin receptor associated kinases (IRAK) 1 and 4 are serine/threonine kinases that are essential for signaling downstream of most TLRs and IL-1Rs and the resulting production of pro-inflammatory cytokines (2). Suppression of TLR and IL-1R signaling through inhibition of IRAK1/4 kinases is a promising therapeutic approach for the treatment of inflammatory and autoimmune diseases.

Objectives: The aim of the study was to characterize the effects of R835, a novel small molecule inhibitor of IRAK1/4, on TLR4 signaling.

Methods: R835 was identified in cell-based assays measuring cytokine production induced by LPS (TLR4 ligand). Inhibition of IRAK1 and IRAK4 kinases by R835 was confirmed in biochemical assays and cell lysates. The ability of R835 to inhibit TLR4 signaling was further evaluated in human and mouse whole blood assays. R835 was tested in a mouse model of LPS-induced cytokine release. Mice were pre-treated orally with vehicle or R835 prior to challenge with LPS and serum cytokine levels were monitored over a 24-hour period.

Results: We have identified R835, a selective small molecule inhibitor of IRAK1 and IRAK4. R835 blocked LPS/TLR4 signaling and the resulting production of proinflammatory cytokines in both human and mouse cells and whole blood. R835 suppressed serum cytokine elevation in mice challenged with LPS.

Conclusion: Our study demonstrates that R835, through inhibition of IRAK1/4 kinase activity, blocks LPS-induced cytokine production in vitro and in vivo. In a recent phase 1 study, R835 substantially reduced the increase of serum cytokines after an intravenous LPS challenge in healthy volunteers. Importantly, this shows that the pharmacological inhibition of IRAK1/4 pathway by R835 in humans mirrors the results obtained in mice. To our knowledge, R835 is the first dual IRAK1/4 inhibitor to enter clinical development and demonstrate inhibition of TLR4-induced cytokines in both mice and humans. R835 is a promising clinical candidate that will allow the exploration of IRAK1/4 inhibition in the treatment of cytokine-driven rheumatic and autoimmune diseases.

References:

[1] The interleukin-1 receptor/ Toll-like receptor superfamily: 10 years of progress. Luke A. J. O'Neill. Immunological Reviews 2008. Vol. 226: 10-18

[2] Flannery S, Bowie A G. The interleukin-1 receptor-associated kinases: Critical regulators of innate immune signaling. Biochemical Pharmacology, Volume 80, Issue 12, 15 December 2010, Pages 1981-1991.

Disclosure of Interests: Chrystelle Lamagna Shareholder of: Rigel Pharmaceuticals, Employee of: Rigel Pharmaceuticals, Caroline Gundel Shareholder of: Shareholder of Rigel Pharmaceuticals, Employee of: Employee of Rigel Pharmaceuticals, Meagan Chan Shareholder of: Rigel Pharmaceuticals,
Employee of: Rigel Pharmaceuticals, Chi Young Shareholder of: Rigel Pharmaceuticals, Employee of: Rigel Pharmaceuticals, Sylvia Braselmann Shareholder of: Shareholder of Rigel Pharmaceuticals, Employee of: Employee of Rigel Pharmaceuticals, Roy Frances Shareholder of: Rigel Pharmaceuticals, Employee of: Rigel Pharmaceuticals, Sothy Yi Shareholder of: Rigel Pharmaceuticals, Employee of: Rigel Pharmaceuticals, Yan Chen Shareholder of: Rigel Pharmaceuticals, Employee of: Rigel Pharmaceuticals, Gary Park Shareholder of: Rigel Pharmaceuticals, Employee of: Rigel Pharmaceuticals, Lu Chou Shareholder of: Rigel Pharmaceuticals, Employee of: Rigel Pharmaceuticals Esteban Masuda Shareholder of: Rigel Pharmaceuticals, Employee of: Rige Pharmaceuticals, Vanessa Taylor Shareholder of: Rigel Pharmaceuticals Employee of: Rigel Pharmaceuticals DOI: 10.1136/annrheumdis-2020-eular.3370

\section{FRI0017 \\ ACTIVATION OF TOLL-LIKE RECEPTORS IN PERIPHERAL BLOOD MONONUCLEAR CELLS OF TAKAYASU ARTERITIS PATIENTS}

Y. Tian ${ }^{1}$, J. Liं ${ }^{2}$, X. Tian ${ }^{2}$, X. Zeng ${ }^{2} .{ }^{1}$ Peking Union Medical College Hospital, Peking Union Medical College and Chinese Academy of Medical Sciences, National Clinical Research Center for Dermatologic and Immunologic Diseases (NCRC-DID), Rheumatology, Beijing, China; ${ }^{2}$ Peking Union Medical College Hospital, Peking Union Medical College and Chinese Academy of Medical Sciences, National Clinical Research Center for Dermatologic and Immunologic Diseases (NCRC-DID), Department of Rheumatology, Beijing, China

Background: The activation of self-specific T cells is essential in pathogenesis of Takayasu arteritis (TAK). Dendritic cell (DC) plays an indispensable role as the only antigen presenting cell for initial $\mathrm{T}$ cell, and Toll-like receptors (TLRs) are common source of activation signals for DCs. Then we speculate that there are activation of TLRs in TAK patients.

Objectives: To investigate the activation of TLRs in TAK patients.

Methods: Twenty-seven TAK patients were enrolled during April to October in 2019, with diagnosis met the 1990 criteria of American College of Rheumatology. Patient were divided into groups by the disease activity and medication history. Disease activity was assessed by the $1994 \mathrm{NIH}$ criteria. Quantitative Real-time Polymerase Chain Reaction (RT-qPCR) was used to analyze the mRNA relative abundance of 28 target genes in peripheral blood mononuclear cells (PBMCs). Differences between groups and correlation between any two genes were analyzed.

Results: The demographic data and clinical features of TAK patients were shown in Table 1. (1) Compared with health control (HC) group, mRNA abundance of TLR2, TLR4, P50, P65, IKBa, CTLA4, CD3, and BCL6 in untreated TAK group was upregulated $(<0.05)$, whereas mRNA abundance of $C D 40$ was downregulated ( $\mathrm{p}<0.05$ ). (2) Compared with HC group, mRNA abundance of TLR2, TLR4, IKBa $P D-1$ and $B C L 6$ in treated TAK group was upregulated $(p<0.05)$, whereas mRNA abundance of $L A G 3, C D 40$ and TCR was downregulated $(p<0.05)$. (3) Compared with untreated TAK group, mRNA abundance of P50, P65, CD28, CTLA4, TLR2, TLR4, IKBa, PD-1 and RORC was upregulated in treated TAK group ( $\mathrm{p}$ $<0.05)$. (4) Compared with non-active treated TAK group, mRNA abundance of p50, CD28, TCR, GATA3, RORC and FOXP3 was upregulated in nonactive treated TAK group $(\mathrm{p}<0.05)$. BCL6 showed correlation with the TLRs-NFKB pathway. (Figure 1 2, Table 2 )

Table 1. Demographic data and clinical features of patients with TAK

\begin{tabular}{|c|c|c|c|c|c|c|c|c|c|}
\hline & \multirow[b]{2}{*}{ Age (year) } & \multirow[b]{2}{*}{$\begin{array}{l}\text { Gender (male/ } \\
\text { female) }\end{array}$} & \multirow[b]{2}{*}{$\begin{array}{l}\text { Disease duration* } \\
\text { (months) }\end{array}$} & \multirow[b]{2}{*}{$\mathrm{ESR}(\mathrm{mm} / \mathrm{h})$} & \multirow[b]{2}{*}{ hs-CRP* (mg/L) } & \multirow[b]{2}{*}{$\begin{array}{l}\text { Interleukin } \\
6(\mathrm{pg} / \mathrm{mL})\end{array}$} & \multirow[b]{2}{*}{$\begin{array}{l}\text { TNFa(pg/ } \\
\mathrm{mL})\end{array}$} & \multicolumn{2}{|c|}{ Prednisone } \\
\hline & & & & & & & & $\begin{array}{c}\text { used/ } \\
\text { non-used }\end{array}$ & Dosage (mg/d) \\
\hline Treated $(n=20)$ & $39.37 \pm 9.27$ & $1 / 19$ & $43(12,103)$ & $14.60 \pm 8.94$ & $1(0.55,5.625)$ & $\begin{array}{l}2.1(2 \\
3.95)\end{array}$ & $7.56 \pm 4.39$ & $18 / 2$ & $10(10,32.5)$ \\
\hline Active $(n=11)$ & $39.30 \pm 7.889$ & $1 / 10$ & $118(16,166.5)$ & $16.82 \pm 10.81$ & $5.63(1.49,8.33)$ & $\begin{array}{l}3.15(2.025, \\
5.775)\end{array}$ & $8.42 \pm 5.57$ & $10 / 1$ & $10(10,15)$ \\
\hline Nonactive $(n=9)$ & $39.44 \pm 10.59$ & $0 / 9$ & $40(12,44)$ & $11.89 \pm 4.61$ & $0.84(0.31,1)$ & $2(2,2.4)$ & $6.60 \pm 2.11$ & $8 / 1$ & $8.75(6.875,16.25)$ \\
\hline$P$ value & 0.89 & - & 0.16 & 0.34 & 0.02 & 0.08 & 0.65 & - & 0.37 \\
\hline \multicolumn{10}{|l|}{ Untreated $(n=7)$} \\
\hline \multicolumn{10}{|l|}{ Active $(n=4)$} \\
\hline 1 & 31 & $M$ & - & 91 & 140.72 & - & - & - & 0 \\
\hline 2 & 25 & $\mathrm{~F}$ & - & 19 & 11.28 & 6.3 & 5.2 & - & 0 \\
\hline 3 & 23 & M & - & 71 & 77.36 & 6.3 & 6.2 & - & 0 \\
\hline 4 & 29 & $\mathrm{~F}$ & - & 127 & 113.62 & 22.2 & 8.4 & - & 0 \\
\hline \multicolumn{10}{|l|}{ Nonactive $(n=3)$} \\
\hline 5 & 34 & $F$ & - & 7 & 0.34 & 2 & 4.3 & - & 0 \\
\hline 6 & 27 & $\mathrm{~F}$ & - & 14 & 0.16 & 25.7 & 4 & - & 0 \\
\hline 7 & 38 & $\mathrm{~F}$ & - & 5 & 0.32 & 3 & 4 & - & 0 \\
\hline
\end{tabular}

* median (min, max) 
Table 2. Genes expressed abnormally in PBMCs of TAK patients

\begin{tabular}{|c|c|c|c|c|c|c|c|c|}
\hline & \multicolumn{2}{|c|}{$\begin{array}{c}\text { Abnormally expressed in } \\
\text { untreated TAK }\end{array}$} & \multicolumn{2}{|c|}{$\begin{array}{c}\text { Abnormally expressed in } \\
\text { treated TAK }\end{array}$} & \multicolumn{2}{|c|}{ Influenced by treatment } & \multicolumn{2}{|c|}{$\begin{array}{l}\text { Associated with the } \\
\text { TAK activity }\end{array}$} \\
\hline & upregulated & downregulated & upregulated & downregulated & upregulated & downregulated & Upregulated & downregulated \\
\hline Genes associated with the TLRs-NFKB pathway & $\begin{array}{l}\text { TLR2, TLR4, p50, } \\
\quad p 65, I_{K B a}\end{array}$ & - & $T L R 2, T L R 4, I K B a$ & - & - & $p 50, p 65$ & p50 & - \\
\hline $\begin{array}{l}\text { Positive and negative costimulatory molecules and } \\
\text { their ligands }\end{array}$ & CTLA4 & $C D 40$ & $P D-1$ & CD40, LAG3 & - & CD28, CTLA4 & $C D 28$ & - \\
\hline $\begin{array}{l}\text { Genes associated with the activation or differentia- } \\
\text { tion of } T \text { cell or } B \text { cell }\end{array}$ & $C D 3, B C L 6$ & - & $B C L 6$ & $T C R$ & - & $C D 3, T C R, R O R C$ & $\begin{array}{l}\text { TCR, GATA3, } \\
\text { RORC, FOXP3 }\end{array}$ & - \\
\hline
\end{tabular}
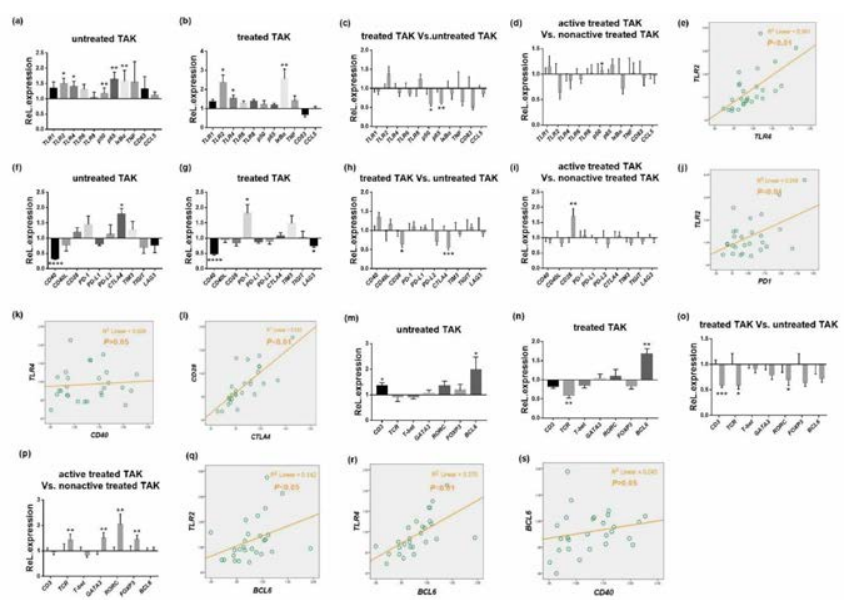

Figure 1. mRNA abundance of genes in PBMCs in TAK patients analyzed by qPCR (a) -(e) Partial genes associated with TLRs-NFKB pathway. (f) -(I) Partial genes associated with the TLRs-NFKB pathway or costimulatory molecules. $(m)-(s)$ Partial genes associated with the activation or differentiation of $T$ cell or $B$ cell. (a)(f)(m) Compare the untreated TAK group with the HC group whose data normalized to one. $(b)(g)(n)$ Compare the treated TAK group with the HC group whose data normalized to one. (c)(h)(o) Compare the untreated TAK group with the treated TAK group whose data normalized to one. (d)(i)(p) Compare the active untreated TAK group with the nonactive treated TAK group whose data normalized to one. (e)(i) - (I)(r) Pearson correlation test, $\mathrm{n}=27$ (all TAK patients enrolled). (q)(s) Spearman correlation test, $\mathrm{n}=27$ (all TAK patients enrolled). Data are mean \pm SEM. Untreated TAK group, $n=7$. Treated TAK group, $n=20$. Active untreated TAK group, $\mathrm{n}=9$. Nonactive treated TAK group, $\mathrm{n}=11 . \mathrm{HC}$ group, $\mathrm{n}=11 ., \quad P<0.05, \cdots, P<0.01, \ldots, p<0.001, \ldots . ., P<0.0001$.

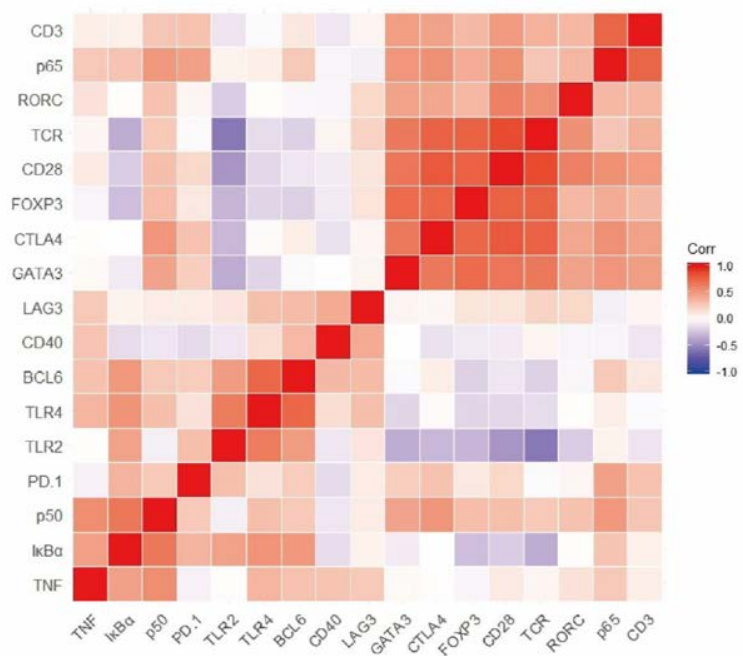

Figure 2 Correlation between any two genes expressed abnormally in PBMCs in TAK patients Cluster Analysis by the result of Spearman correlation test. $n=27$ (all TAK patients enrolled).

Conclusion: TLRs-NFKB pathway may be activated in TAK patients, with upregulation of BCL6, and there may be deficiency of CD40. TLR2, TLR4, $P D-1, L A G 3, C D 40$ and BCL6 may play roles in the pathogenesis of TAK. $p 50$, CD28, TCR, GATA3, RCRC and FOXP3 may be related to the disease activity of TAK.

Disclosure of Interests: Yixiao Tian: None declared, Jing Li: None declared, Xinping Tian: None declared, Xiaofeng Zeng Consultant of: MSD Pharmaceuticals DOI: 10.1136/annrheumdis-2020-eular.4161

\section{FRIDAY, 05 JUNE 2020 \\ Rheumatoid arthritis - prognosis, predictors and outcome}

\begin{tabular}{|l|l|}
\hline FRI0018 & USING SELF-REPORTED OUTCOMES TO DETECT \\
NEW-ONSET FLARE IN A REAL-WORLD STUDY OF \\
PARTICIPANTS WITH RHEUMATOID ARTHRITIS - \\
INTERIM RESULTS FROM THE DIGITAL TRACKING OF \\
ARTHRITIS LONGITUDINALLY (DIGITAL) STUDY
\end{tabular}

V. S. Haynes ${ }^{1}$, J. Curtis², F. Xie ${ }^{2}$, I. Lipkovich', H. Zhao ${ }^{2}$, C. L. Kannowski', J. L. Poon ${ }^{1}$, K. Gavigan ${ }^{3}$, D. Curtis ${ }^{3}$, S. K. Nolot ${ }^{1}$, W. B. Nowell ${ }^{3}{ }^{1}$ Eli Lilly and Company, Indianapolis, United States of America; ${ }^{2}$ University of Alabama at Birmingham, Birmingham, United States of America; ${ }^{3}$ Global Healthy Living Foundation, Upper Nyack, United States of America

Background: Patients with rheumatoid arthritis (RA) experience fluctuating symptoms, increased pain, decreased function and variable quality of life; such changes often occur between visits to clinicians. Digital Tracking of Arthritis Longitudinally (DIGITAL) study ${ }^{2}$ is evaluating the use of electronically captured patient-reported outcomes (ePRO) and passive data collection from a Fitbit device to identify disease worsening in a real-world study of participants (pts) with RA.

Objectives: Evaluate agreement between self-reported new-onset flare and ePROs in an interim analysis from DIGITAL using a classification model.

Methods: Members of the ArthritisPower registry with RA were invited to participate in DIGITAL. Pts who successfully completed a two-week Lead-in period entered the Main Study in which they wore a smartwatch and provided daily (pain and fatigue numeric rating scales (NRS)) and weekly ePROs, including the OMERACT RA Flare Questionnaire (FLARE) and PROMIS measures. This interim analysis is of ePRO data from pts who completed at least 30 days of the Main Study. A "Yes" response to the FLARE item, "Are you having a flare now?" identified flare. For modeling association between new-onset flare and ePRO, the dataset was split into training (the first 30 days of the Main Study) and test data (Day 31 and following). Within each dataset, repeated binary outcomes (Flare/No Flare) per pt were defined each week. To focus on new-onset flare, within each dataset, outcomes for patient weeks for which flare was present in the previous week were excluded.

Candidate variables for the model included baseline and current FLARE score (0-50 scale) and each of its 5 items, daily pain, daily fatigue, and several PROMIS weekly instruments and their lagged values (last week or last 6 days for daily). 'Baseline' was calculated in non-flare weeks. Training data was used for logistic regression model selection combining clinical expertise with backward elimination. Performance of the final model was evaluated using test data.

Results: The training data was composed of outcomes from 128 pts who reported 388 weekly flare assessments as no flare or onset flare over 2800 days during the first month of the Main Study. Of pts in the training dataset, $92.2 \%$ were female, 87.5\% white, with mean age (SD) 52.7 (11.0) and years since RA diagnosis 10.4 (10.3); $62.5 \%$ were on a biologic. Among those in the training dataset, 58 flare outcomes occurred in $50(39.1 \%)$ unique pts.

The test data comprised outcomes from 123 pts who reported 442 weekly flare assessments as no flare or onset flare over 3366 days in which 64 flare outcomes occurred, and primarily included continued observations from pts who contributed to the training dataset.

The best-performing model to classify flare in training data included the current and baseline FLARE instrument activity question (i.e. "Considering how active your rheumatoid arthritis has been, how much difficulty have you had when taking part in activities such as work, family life, social events that are typical for you during the last week"), current daily pain, and baseline daily pain average and standard deviation. In test data, this model had an area under the receiver operator curve of 0.81 (Figure). At a cut point requiring specificity to be $\geq 0.80$, sensitivity to detect flare was 0.62 and overall accuracy was 0.78 . 